\title{
External Ear Features: Role in Tracing Inheritance
}

\author{
Bhima Neupane, ${ }^{1}$ Kanaklata lyer, ${ }^{1}$ Chachhu Bhattarai, ${ }^{1}$ Brihaspati sigdel ${ }^{2}$ \\ 'Department of Anatomy, Manipal College of Medical Sciences, Pokhara, Nepal ${ }^{2}$ Department of Otolaryngology \& \\ Head and Neck Surgery, Gandaki Medical College, Pokhara, Nepal
}

\section{ABSTRACT}

\section{Introduction}

The external ear is unique in shape, size and orientation. It has symmetry and asymmetry among generations. Its morphological variation helps to trace genetic inheritance. The objective of this study was to identify this morphological variation among parents and siblings.

\section{Methods}

The study was conducted in Manipal colleges of medical sciences. Images were procured from 147 families. The morphological character of external ears including, shape of pinnae, concha, tragus, ear lobule and attachment of lobule to cheek were matched among parents and offspring.

\section{Results}

This study included 882 pinnae in 147 families. Total 4410 morphological characteristics have been analyzed. Tragus was the most common matching character between parents and offspring Kappa measuring coefficient was statistically significant for shape of pinna, concha, ear lobule, ear lobule attachment to cheek.

\section{Conclusions}

The most of external ear morphology matches between parents and offspring which may be a helpful tool in tracing hereditary inheritance.

Keywords: external ear morphology; genetic inheritance; matching character.

\section{INTRODUCTION}

The use of ear as a tool of human identification began in the late 19th century. ${ }^{1}$ Entire pinnae is a single piece of fibrocartilaginous structure having irregular surface with varying degree of elevations and depressions. ${ }^{2}$ The morphological character of the external ear has a unique character in every individual. ${ }^{3}$

Due to its unique feature, it is used in personal identification by many scientists. It is a fact that various features of the external ear are under multiple genetic controls and are expected to behave in a similar manner in genetically related persons. ${ }^{4}$ It was therefore considered important

Correspondance: Dr. Bhima Neupane, Department of Anatomy, Manipal College of Medical Sciences, Pokhara, Nepal, Email: bhimasigdel@gmail.com, Phone no: 9779846412100. 
to evaluate its degree of similarities among generations. ${ }^{5}$

The aim of this study is to observe the external ear features such as shape of pinna, tragus, concha, ear lobule and its attachment to cheek and to find out whether there is similarities between parents and offspring from above mention structures.

\section{METHODS}

The study was conducted in Manipal colleges of medical sciences. Informed \& written consent was taken from all subjects. Ethical clearance done from Institutional review board of Manipal College of Medical Science (MCOMSs), Pokhara, Nepal. Data was collected from the family of Nepali students, staff, and patient's visitors from MCOMS and Manipal Teaching Hospital from $1^{\text {st }}$ January 2020 to $30^{\text {th }}$ July 2020.

The study covered all cross sections of people. In total, images were procured from 147 families. The final sample for study includes families of two generations.

All the subjects were normal and healthy. None of them were suffering from any auricular (congenital and traumatic) or maxillofacial deformity or had undergone any auricular surgical procedure.

Bilateral images of subjects were taken from a distance of $0.5 \mathrm{~m}$ from Samsung Pro android mobile with a 12 pixel rear camera. During photography the head of the subject was oriented in the Frankfort horizontal plane and the focal plane of the camera was parallel to the longitudinal plane of the external ear. The camera was fixed on a tripod so that it could be elevated to the level of ear of the subject. The images were acquired in daylight. The parts of the external ear studied are shape of Pinnae concha, tragus, ear lobule and its attachment to check in both right and left pinnae (Figure 1 and Figure 2). Somatoscopic features of the ears of father, mother and first offspring were categorized according to their character. Morphological features of external ear include shape of pinnae, concha, tragus ear lobule and ear lobule attachment to cheek. Pinnae morphology of the same side were matched between parent and offspring. Matching pairs were calculated in percentage. Measurement of agreement was performed by Kappa Coefficient and association by chi-square on SPSS software 26.0 version. Value of agreement or association less than 0.05 was statistically significant.

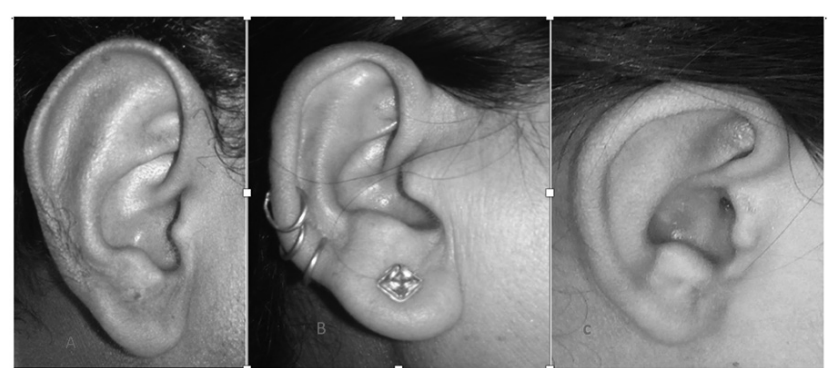

Figure 1. Showing right pinnae: A. father B. mother c. daughter

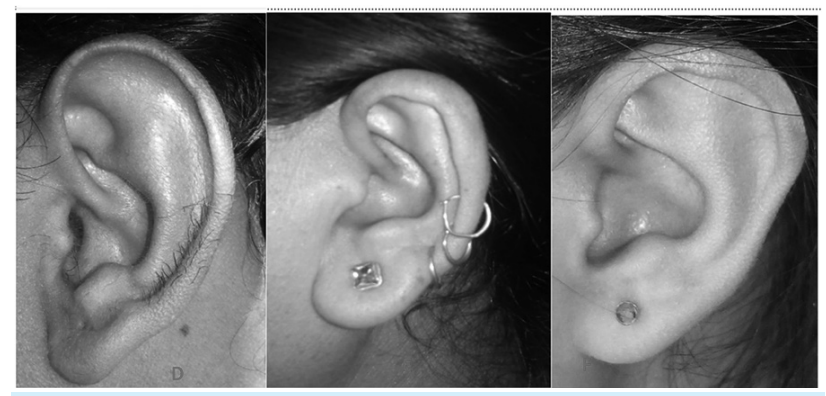

Figure 2. Showing left pinnae: D. father E. mother F. daughter

\section{RESULTS}

In this study we includes 882 pinnae of 147 families. Total 4410 morphological characteristics have been analyzed. Each feature were classified into its sub-character. In total we observe 14994 distinct sub-characters.

Tragus was the most common matching character found between parent and offspring [Right father versus offspring: 104(70.7\%) and left father versus offspring 106(72.1\%)]. The most common character in both right and left ears was round-round 63 (42.8\%). Kappa measuring coefficient was statistically significant for pinna 
shape, concha shape, ear lobule shape, ear lobule attachment to cheek. (Table 1 and Table 2)
When comparing mother pinnae and offspring, characteristics such as shape of

Table 1. somatoscopic analysis showing father and offspring pair matching character, Degree of Association $\left(\chi^{2}\right)$ and measurement of agreement (Kappa coeff $\left.=\kappa\right)$ of Right and left pinnae.

\begin{tabular}{|c|c|c|c|c|c|c|}
\hline Morphology & Side of ear & $\begin{array}{l}\text { Pair matching } \\
\text { character }\end{array}$ & matching $(\%)$ & Total & $\begin{array}{c}\text { Chi-square }\left(\chi^{2}\right) \\
\text { and } \kappa \text {-value }\end{array}$ & $P$ value \\
\hline \multirow{8}{*}{ pinnae shape } & \multirow{4}{*}{ Right } & Oval-Oval & $30(20.4 \%)$ & \multirow{4}{*}{$63(42.8 \%)$} & \multirow{4}{*}{$\chi^{2}=32.68$} & \multirow{4}{*}{0.001} \\
\hline & & Rectang-Rectang* & $11(7.4 \%)$ & & & \\
\hline & & Round-Round & $11(7.4 \%)$ & & & \\
\hline & & Triang- Triang $\dagger$ & $11(7.4 \%)$ & & & \\
\hline & \multirow{4}{*}{ Left } & Oval-Oval & $26(17.6 \%)$ & \multirow{4}{*}{$64(43.5 \%)$} & \multirow{4}{*}{$\chi^{2}=35.6$} & \multirow{4}{*}{0.000} \\
\hline & & Rectang-Rectang* & $15(10.2 \%)$ & & & \\
\hline & & Round-Round & $13(8.8 \%)$ & & & \\
\hline & & Triang- Triang $\dagger$ & $10(6.8 \%)$ & & & \\
\hline \multirow{6}{*}{ Concha shape } & \multirow{3}{*}{ Right } & Broad-Broad & $5(3.4 \%)$ & \multirow{3}{*}{$81(55.1 \%)$} & \multirow[t]{3}{*}{$\chi^{2}=7.83$} & \multirow[b]{3}{*}{0.074} \\
\hline & & Narrow-Narrow & $7(4.7 \%)$ & & & \\
\hline & & Proport- Proport $\ddagger$ & $69(68.5 \%)$ & & & \\
\hline & \multirow{3}{*}{ Left } & Broad-Broad & $9(6.1 \%)$ & \multirow{3}{*}{$95(64.6 \%)$} & \multirow[t]{3}{*}{$\chi^{2}=35.2$} & \multirow[t]{3}{*}{0.000} \\
\hline & & Narrow-Narrow & $9(6.1 \%)$ & & & \\
\hline & & Proport- Proport $\ddagger$ & $77(52.3 \%)$ & & & \\
\hline \multirow{6}{*}{ Tragus shape } & \multirow{3}{*}{ Right } & Knob-Knob & $34(23.1 \%)$ & \multirow{3}{*}{$104(70.7 \%)$} & \multirow{3}{*}{$\chi^{2}=75.3$} & \multirow[t]{3}{*}{0.00} \\
\hline & & Long-Long & $7(4.7 \%)$ & & & \\
\hline & & Round-Round & $63(42.8 \%)$ & & & \\
\hline & \multirow{3}{*}{ Left } & Knob-Knob & $38(25.8 \%)$ & \multirow{3}{*}{$106(72.1 \%)$} & \multirow{3}{*}{$\chi^{2}=72.8$} & \multirow[t]{3}{*}{0.00} \\
\hline & & Long-Long & $5(3.4 \%)$ & & & \\
\hline & & Round-Round & $63(42.8 \%)$ & & & \\
\hline
\end{tabular}

*Rectang-Rectang:Rectangular-Rectangular, †Triang- Triang:Triangular:-Triangular $\ddagger$ Proport- Proport: proportionate-proportionate

Table 2. somatoscopic analysis showing father and offspring pair matching character, Degree of Association $\left(\chi^{2}\right)$ and measurement of agreement (Kappa coeff $=\kappa$ ) of Right and left ear lobule shape and its attachment to cheek.

\begin{tabular}{|c|c|c|c|c|c|c|}
\hline Morphology & Side of ear & $\begin{array}{c}\text { Pair matching } \\
\text { character }\end{array}$ & matching (\%) & Total & $\begin{array}{c}\text { Chi-square }\left(\chi^{2}\right) \\
\text { and } \kappa \text {-value }\end{array}$ & $P$ value \\
\hline \multirow{5}{*}{$\begin{array}{l}\text { Ear lobule } \\
\text { shape }\end{array}$} & \multirow[t]{5}{*}{ Right } & Arched-Arched & $19(12.9 \%)$ & \multirow{5}{*}{$66(44.9 \%)$} & \multirow[t]{5}{*}{$\chi^{2}=64$} & \multirow[t]{5}{*}{0.00} \\
\hline & & Rectang-Rectang* & $7(4.7 \%)$ & & & \\
\hline & & Round-round & $23(15.6 \%$ & & & \\
\hline & & Tongue-tongue & $14(9.5 \%)$ & & & \\
\hline & & Triang-triang $\dagger$ & $3(2.1 \%)$ & & & \\
\hline
\end{tabular}




\begin{tabular}{|c|c|c|c|c|c|c|}
\hline & \multirow[t]{5}{*}{ Left } & Arched-arched & $20(13.6 \%)$ & \multirow[t]{5}{*}{$74(50.3 \%)$} & \multirow{5}{*}{$\begin{array}{l}\chi^{2}=99.02 \\
\kappa=0.348\end{array}$} & \multirow[t]{5}{*}{0.000} \\
\hline & & Rectang-Rectang* & $6(4.0 \%) \dagger$ & & & \\
\hline & & Round-round & $31(21.0 \%)$ & & & \\
\hline & & Tongue-tongue & $13(8.8 \%)$ & & & \\
\hline & & Triang-triang $\dagger$ & $4(2.7 \%)$ & & & \\
\hline \multirow{6}{*}{$\begin{array}{l}\text { Ear lobule } \\
\text { attachment } \\
\text { to cheek }\end{array}$} & \multirow[t]{3}{*}{ Right } & Free-Free & $34(23.1 \%)$ & \multirow[t]{3}{*}{$76(51.7 \%)$} & \multirow[t]{3}{*}{$\chi^{2}=22.15$} & \multirow[t]{3}{*}{0.000} \\
\hline & & Part att-Part att§ & $28(19.0 \%)$ & & & \\
\hline & & Attached-Attached & $14(9.5 \%)$ & & & \\
\hline & \multirow[t]{3}{*}{ Left } & Free-Free & $32(21.7 \%)$ & \multirow[t]{3}{*}{$72(48.9 \%)$} & \multirow[t]{3}{*}{$\chi^{2}=20.01$} & \multirow[t]{3}{*}{0.018} \\
\hline & & Part att-Part att $\S$ & $26(17.6 \%)$ & & & \\
\hline & & Attached-Attached & $14(9.5 \%)$ & & & \\
\hline
\end{tabular}

*Rectang-Rectang:Rectangular-Rectangular, †Triang- Triang:Triangular:-Triangular †Proport- Proport: proportionate-proportionate

pinnae, concha, tragus and ear lobule have been found to be substantially matched with ( $p$ value $<0.05$ ).In parents and offspring the most prominent pinnae form was oval-oval.
Comparing the right lobule attachment, free lobule was more common. Fathers ear were more matched than mothers $(34 \%$ versus $21.7 \%$ ) (Table 3 and 4 ).

Table 3. somatoscopic analysis showing mother and offspring pair matching character, Degree of Association $\left(\chi^{2}\right)$ and measurement of agreement (Kappa Coeff. $=\kappa$ ) of Right and left shape of pinnae, concha and tragus.

\begin{tabular}{|c|c|c|c|c|c|c|}
\hline $\begin{array}{l}\text { Morphology } \\
\text { of pinnae }\end{array}$ & $\begin{array}{l}\text { Side } \\
\text { of ear }\end{array}$ & $\begin{array}{l}\text { Pair matching } \\
\text { character }\end{array}$ & $\begin{array}{c}\text { matching } \\
(\%)\end{array}$ & Total & $\begin{array}{c}\text { Chi-square }\left(\chi^{2}\right) \\
\text { and } \kappa \text { coefff }\end{array}$ & $P$ value \\
\hline \multirow[t]{8}{*}{ pinnae shape } & \multirow[t]{4}{*}{ Right } & Oval-Oval & $27(18.3 \%)$ & \multirow[t]{4}{*}{$54(36.7 \%)$} & \multirow{4}{*}{$\begin{array}{l}\chi^{2}=34.21 \\
\kappa=0.205\end{array}$} & \multirow{4}{*}{$\begin{array}{l}0.001 \\
0.000\end{array}$} \\
\hline & & Rectang-Rectang* & $11(7.5 \%)$ & & & \\
\hline & & Round-Round & $16(10.8 \%)$ & & & \\
\hline & & Triang- Triang $\dagger$ & $7(4.7 \%)$ & & & \\
\hline & \multirow[t]{4}{*}{ Left } & Oval-Oval & $28(19.0 \%)$ & \multirow[t]{4}{*}{$59(40.1 \%)$} & \multirow{4}{*}{$\begin{array}{l}\chi^{2}=36.84 \\
\kappa=0.183\end{array}$} & \multirow{4}{*}{$\begin{array}{l}0.002 \\
0.000\end{array}$} \\
\hline & & Rectang-Rectang* & $15(10.2 \%)$ & & & \\
\hline & & Round-Round & $9(6.1 \%)$ & & & \\
\hline & & Triang- Triang $\dagger$ & $7(4.7 \%)$ & & & \\
\hline \multirow[t]{6}{*}{ Concha shape } & \multirow[t]{3}{*}{ Right } & Broad-Broad & $4(2.7 \%)$ & \multirow[t]{3}{*}{$86(58.5 \%)$} & \multirow{3}{*}{$\begin{array}{l}\chi^{2}=16.14 \\
\kappa=0.204\end{array}$} & \multirow{3}{*}{$\begin{array}{l}0.003 \\
0.001\end{array}$} \\
\hline & & Narrow-Narrow & $13(9.0 \%)$ & & & \\
\hline & & Proport- Proport $\ddagger$ & $69(46.9 \%)$ & & & \\
\hline & \multirow[t]{3}{*}{ Left } & Broad-Broad & $2(1.3 \%)$ & \multirow[t]{3}{*}{$89(60.5 \%)$} & \multirow{3}{*}{$\begin{array}{l}\chi^{2}=21.69 \\
\kappa=0.200\end{array}$} & \multirow{3}{*}{$\begin{array}{l}0.001 \\
0.001\end{array}$} \\
\hline & & Narrow-Narrow & $13(8.8 \%)$ & & & \\
\hline & & Proport- Proport & $74(50.3 \%)$ & & & \\
\hline \multirow[t]{6}{*}{ Tragus shape } & \multirow{3}{*}{ Right } & Knob-Knob & $32(21.7 \%)$ & \multirow[t]{3}{*}{$105(71.4 \%)$} & \multirow[t]{3}{*}{$\chi^{2}=59.14$} & \multirow[t]{3}{*}{0.00} \\
\hline & & Long-Long & $3(2.0 \%)$ & & & \\
\hline & & Round-Round & $70(47.6 \%)$ & & & \\
\hline & \multirow[t]{3}{*}{ Left } & Knob-Knob & $38(25.8 \%)$ & \multirow[t]{3}{*}{$111(75.5 \%)$} & \multirow[t]{3}{*}{$\chi^{2}=78.31$} & \multirow[t]{3}{*}{0.00} \\
\hline & & Long-Long & $3(2.0 \%)$ & & & \\
\hline & & Round-Round & $70(47.6 \%)$ & & & \\
\hline
\end{tabular}

*Rectang-Rectang: Rectangular-Rectangular, †Triang- Triang:Triangular:-Triangular $\ddagger$ Proport- Proport: proportionate-proportionate 
Table 4. somatoscopic analysis showing mother and offspring pair matching character, Degree of Association $\left(\chi^{2}\right)$ and measurement of agreement (Kappa Coeff. $=k$ ) of Right and left ear lobule shape and its attachment to cheek.

\begin{tabular}{|c|c|c|c|c|c|c|}
\hline $\begin{array}{c}\text { Morphology } \\
\text { of pinnae }\end{array}$ & $\begin{array}{l}\text { Side } \\
\text { of ear }\end{array}$ & $\begin{array}{c}\text { Pair matching } \\
\text { character }\end{array}$ & $\begin{array}{c}\text { matching } \\
(\%)\end{array}$ & Total & $\begin{array}{c}\text { Chi-square }\left(\chi^{2}\right) \\
\text { and } \kappa \text { coeff }\end{array}$ & $P$ value \\
\hline \multirow{10}{*}{$\begin{array}{c}\text { Ear lobule } \\
\text { shape }\end{array}$} & \multirow[t]{5}{*}{ Right } & Arched-Arched & $15(10.2 \%)$ & \multirow[t]{5}{*}{$57(38.7 \%)$} & \multirow{5}{*}{$\begin{array}{l}\chi^{2}=35.2 \\
\kappa=0.20\end{array}$} & \multirow{5}{*}{$\begin{array}{l}0.004 \\
0.00\end{array}$} \\
\hline & & Rectang-Rectang* & $5(3.4 \%)$ & & & \\
\hline & & Round-round & $25(17.0 \%)$ & & & \\
\hline & & Tongue-tongue & $10(6.8 \%)$ & & & \\
\hline & & Triangle-triangle & $2(1.3 \%)$ & & & \\
\hline & \multirow[t]{5}{*}{ Left } & Arched-arched & $14(9.5 \%)$ & \multirow[t]{5}{*}{$66(44.8 \%)$} & \multirow{5}{*}{$\begin{array}{l}\chi^{2}=56.6 \\
\kappa=0.266\end{array}$} & \multirow{5}{*}{$\begin{array}{l}0.000 \\
0.000\end{array}$} \\
\hline & & Rectang-Rectang* & $5(3.4 \%)$ & & & \\
\hline & & Round-round & $33(22.4 \%)$ & & & \\
\hline & & Tongue-tongue & $12(8.1 \%)$ & & & \\
\hline & & Triang-triang $†$ & $2(1.3 \%)$ & & & \\
\hline \multirow{6}{*}{$\begin{array}{c}\text { Ear lobule } \\
\text { attachment } \\
\text { cheek }\end{array}$} & \multirow[t]{3}{*}{ Right } & Free-Free & $31(21.0 \%)$ & \multirow[t]{3}{*}{$74(50.3 \%)$} & \multirow[t]{3}{*}{$\chi^{2}=22.72$} & \multirow[t]{3}{*}{0.000} \\
\hline & & Part att-Part att§ & $22(14.9 \%)$ & & & \\
\hline & & Attached-Attached & $21(14.2 \%)$ & & & \\
\hline & \multirow[t]{3}{*}{ Left } & Free-Free & $30(20.4 \%)$ & \multirow[t]{3}{*}{$72(48.9 \%$} & \multirow[t]{3}{*}{$\chi^{2}=22.90$} & \multirow[t]{3}{*}{0.006} \\
\hline & & Part att-Part att $\S$ & $22(14.9 \%)$ & & & \\
\hline & & Attached-Attached & $20(13.6 \%)$ & & & \\
\hline
\end{tabular}

*Rectang-Rectang:Rectangular-Rectangular, †Triang- Triang:Triangular:-Triangular \$Proport- Proport: proportionate-proportionate, §Part att-Part att:partially attached-partially attached.

\section{DISCUSSION}

The human external ear displays extremely complex shape and symmetry. It has interindividual variation which is unique for each person. ${ }^{6}$ Some forms of human ear shape variation have been known for over a century and were used by physiognomists of the 19th century. ${ }^{7}$ We studied matching symmetry in the human pinnae among parents and offspring from view of anatomical perspective. Pinnae has unique characters which can be used for personal identification and similarity among vertical generations. ${ }^{8}$

We studied different external ear characters among parents and offspring and compared with their offspring. On this study, Oval shaped pinnae was found to be the commonest among parents and offspring.

Tragus was found to be the most common matching followed by concha. Round type of tragus was commonly seen followed by knob type. Our study showed more than two third tragus matched morphologically.

While studying attachment of lobule, free lobule were found to be commonest among parents and offspring. In this study, we also found that $50 \%$ of the population have similar matching of ear lobules. Bhasin mentioned that, attachment to the ear lobule has been found to be an important marker in population genetics. ${ }^{9}$ Male 
and female offspring inherited the trait from both mothers and father irrespective of sex. ${ }^{10}$ This is in agreement with a study done by Hays F et.al. when he crossed Rhodes island red and leghorns. ${ }^{11}$ Free ear lobule were also commonly found in study carried out by Singh $\mathrm{P}$ et al ${ }^{12}$.In Nigerian families, attached and detached (free) ear lobes of the offspring were in the ratio of 1:3. While observing inheritance pattern of earlobe attachment amongst Nigerian families, when both parents have detached earlobes, 160 $(59.93 \%)$ of their offspring had detached earlobes whereas 22 (23.65\%) had attached earlobes which is highly significant. ${ }^{10}$ While observing Korean study, overall attached type of earlobe was more frequent than free type of earlobe. Attached type of ear lobe is more common in both sexes in Korean population. ${ }^{13}$

Overall, External ear characteristics are an important feature not only for personal

\section{REFERENCES}

1. Dhanda V, Badhan JS and Garg RK. Studies on the development of latent ear prints and their significance in personal identification. Problems of Forensic Sciences. 2011; 88: 285-295. http://www. forensicscience.pl/pfs/88_Garg.pdf

2. Sforza C, Grandi G, Binelli M, Tommasi DG, Rosati R, Ferrario VF. Age-and sexrelated changes in the normal human ear. Forensic science international. 2009 May 30;187(1-3):110-e. https://doi. org/10.1016/j.forsciint.2009.02.019

3. Krishan K, Kanchan $\mathrm{T}$ and Thakur S. A study of morphological variations of the human ear for its applications in personal identification. Egyptian Journal of Forensic Sciences. 2019; 9: 6. DOI: 10.1186/s41935-019-0111-0. identification but also for tracing genetic association among family members. Limitation of this study is sample size of this study is small and we have taken picture in 2D photographs. It would be better if the study is carried out in larger population with a $3 \mathrm{D}$ picture.

\section{CONCLUSIONS}

Matching symmetry was observed in all anatomical sub-structures of the external ear. Tragus and concha have almost two-third matching among parents and offspring. Pinnae and lobule had fairly equal levels of symmetry and asymmetry. So from this study we can conclude that there is similarities in ear features between parents and offspring while observing morphological feature of external ear

Conflict of interest: None.
4. Purkait R. Application of external ear in personal identification: a somatoscopic study in families. Ann Forensic Res Anal. 2015; 2: 1015. https://www.jscimedcentral. com/Forensic/forensic-2-1015.pdf

5. Purkait R. External ear: An analysis of its uniqueness. Egyptian Journal of Forensic Sciences. 2016; 6: 99-107. https://doi. org/10.1016/j.ejfs.2016.03.002

6. Claes P, Reijniers J, Shriver MD, et al. An investigation of matching symmetry in the human pinnae with possible implications for 3D ear recognition and sound localization. J Anat. 2015; 226: 6072. 2014/11/11. DOI: 10.1111/joa.12252.

7. Beard JS. The physiognomic approach. Classification of plant communities. Springer. 1978, pp.33-64. https://doi. org/10.1007/978-94-009-9183-5_2 
8. Verma $P$, Sandhu HK, Verma KG, et al. Morphological Variations and Biometrics of Ear: An Aid to Personal Identification. J Clin Diagn Res. 2016; 10: Zc138-142. 2016/07/21. DOI: 10.7860/ jcdr/2016/18265.7876.

9. Bhasin M. Ear lobe attachment among the Newars of Nepal. Human Heredity. 1969: 506-508. PMID: 5365889,DOI: 10.1159/000152259

10. Ordu K, Didia B and Egbunefu $\mathrm{N}$. Inheritance pattern of earlobe attachment amongst Nigerians. Greener Journal of Human Physiology and
Anatomy 2014; 2: 1-7. DOI: 10.15580/ GJHPA.2014.1.012214054

11. Hays F. Inheritance of mottled earlobes and stubs in Rhode Island Reds. The American Naturalist .1943; 77: 471-475. DOI:10.1086/281149

12. Singh P and Purkait R. Observations of external ear--an Indian study. Homo 2009; 60: 461-472. 2009/09/15. DOI: 10.1016/j.jchb.2009.08.002.

13. Kim K, Song W and Kim D. Reevaluation of the earlobe types in Koreans. HOMO 2018; 69: 377-380. PMID: 30392739 DOI: $10.1016 /$ j.jchb.2018.10.003

Citation: Neupane B, lyer K, Bhattarai C, Sigdel B. External Ear Features: Role in Tracing Inheritance. JCMS Nepal. 2020; $16(4): 201-7$. 